Supporting Information

\title{
Absorption Features in CdTe Nanoclusters: \\ Aspect Ratio Dependency of the Singlet/Doublet from First-Principles Calculations
}

\author{
Mei Liu ${ }^{\dagger}$, Yongcheng $\mathrm{Zhu}^{\dagger}$, Xiaolin Wang ${ }^{\dagger}$, Gang Jiang ${ }^{\dagger}$, \\ Chaoran Luan", ${ }^{*}$, Kui Yu, ${ }^{*,+, \#}$ \\ 'Institute of Atomic and Molecular Physics, Sichuan University, \\ 610065 Sichuan, P. R. China \\ "Laboratory of Ethnopharmacology, West China School of Medicine, \\ West China Hospital, Sichuan University, 610065 Sichuan, P. R. China \\ \#Engineering Research Center in Biomaterials, Sichuan University, \\ 610065 Sichuan, P. R. China
}

C. L. (email: luanc@scu.edu.cn) or to K. Y. (email: kuiyu@scu.edu.cn)

\section{Table of Contents}

Figure S1 Comparison of spectra for $\mathrm{Cd}_{37} \mathrm{Te}_{20} \mathrm{H}_{68}^{\prime}$ and CdTe sMSC-371.

Figure S2 Calculated absorption spectra of $\mathrm{Cd}_{37} \mathrm{Te}_{20}$ (a7 to a9) with pseudo hydrogens.S3

Figure S3 Comparison of calculated spectra and absorption cross section predicted from Mie-Gans.

Table S1 The values of depolarization factor $G_{i}$ for all these clusters. S5

Figure S4 Dielectric function of nanoclusters and zinc-blende CdTe. S6

Table S2 Square of TDM $\left(\mu^{2}\right)$ and oscillator strengths $(f)$ for CdTe nanoclusters. $\quad$ S7

Figure S5 Energy level diagrams for all these clusters. S9

Table S3 The first twenty results of $\mu^{2}$ and $f$ in a3 and a4. S10

Figure S6 Density of states for CdTe nanoclusters.

REFERENCES 


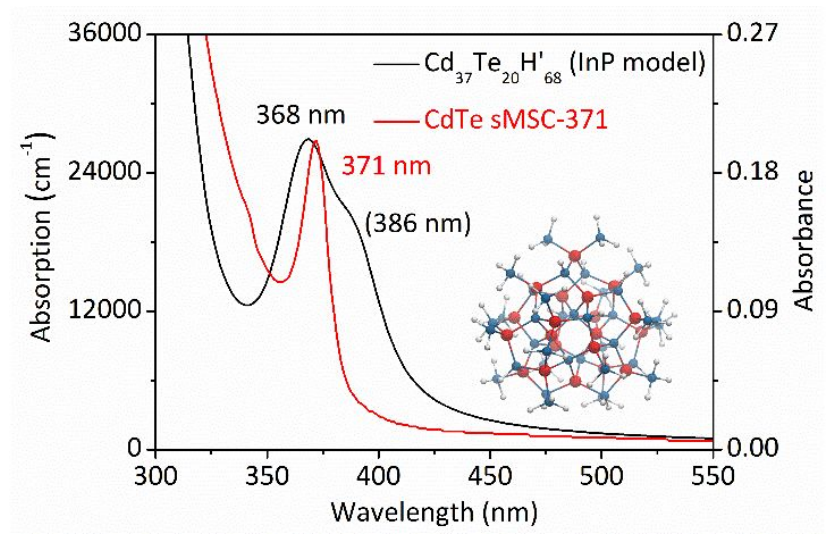

Figure S1. Calculated absorption spectra of $\mathrm{Cd}_{37} \mathrm{Te}_{20}\left(\operatorname{InP}\right.$ model $\left.{ }^{1}\right)$ with partially charged fictitious pseudo hydrogens (black trace), which are compared with the absorption of CdTe sMSC-371 in the experiment ${ }^{2}$ (red trace). The blue balls represent $\mathrm{Cd}$ atoms, the red balls represent Te atoms and the white balls represent $\mathrm{H}^{\prime}$ atoms. 

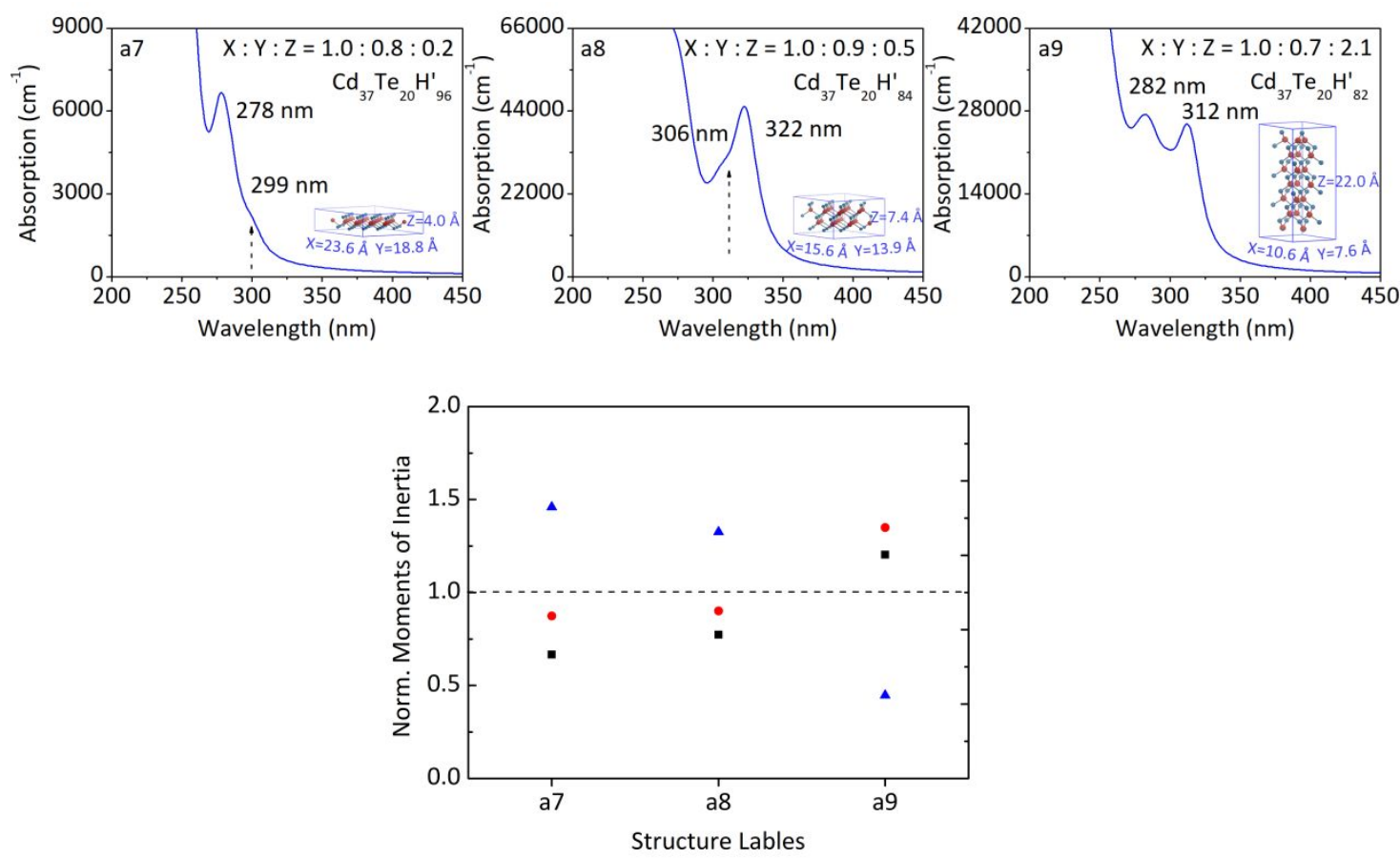

Figure S2. (top) Calculated absorption spectra of $\mathrm{Cd}_{37} \mathrm{Te}_{20}$ (a7 to a9) with partially charged fictitious pseudo hydrogens. The insets show the corresponding atomic structures and length of three dimensions for core clusters. The blue balls represent Cd atoms and the red balls represent Te atoms. (bottom) Normalized moments of inertia $\left\langle l_{i}\right\rangle$ for core clusters. The moments relative to $x, y, z$ axes are represented by black, red, and blue dots, respectively. 

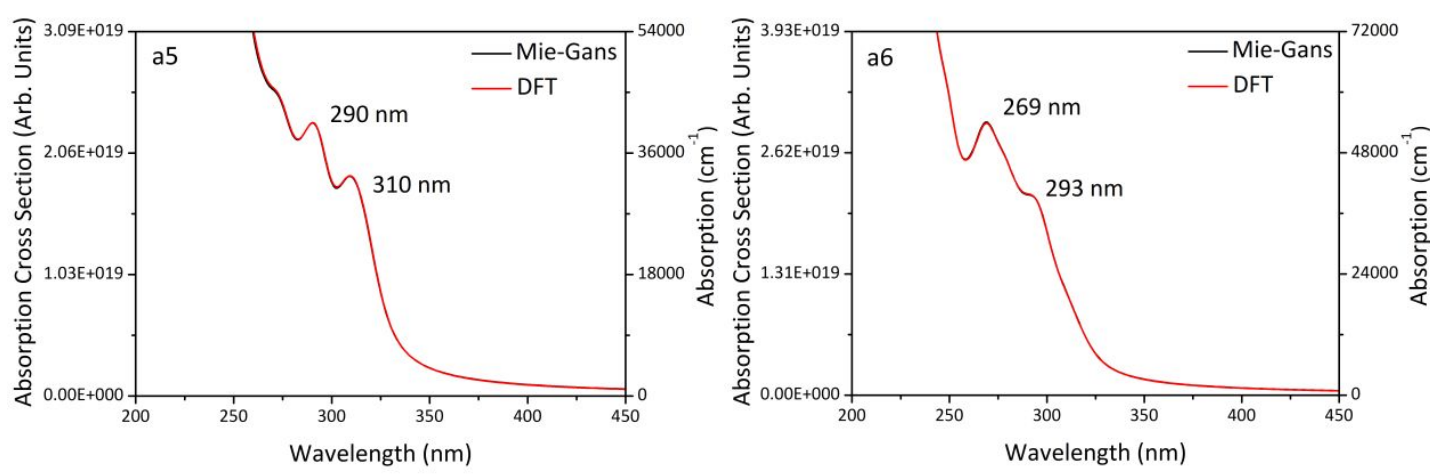

(a)
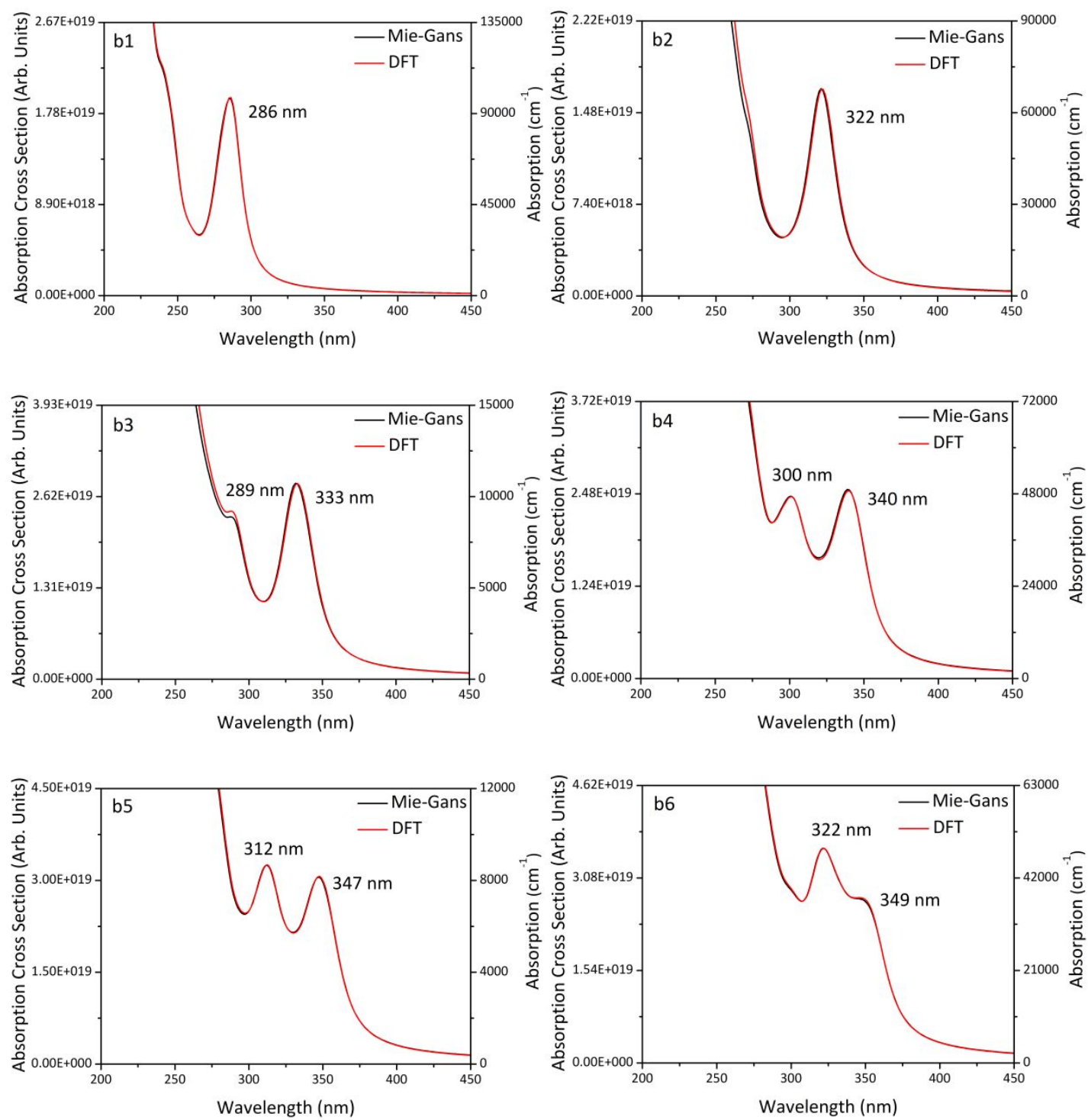

(b)

Figure S3. Predictions from Mie-Gans theory for the absorption cross section per atom as a function of wavelength for (a) $\mathrm{Cd}_{37} \mathrm{Te}_{20}$ isomers (b) CdTe non-isomers, which are compared with DFT absorption spectra. 
Table S1. The values of depolarization factor $G_{i}$ for (a) $\mathrm{Cd}_{37} T \mathrm{Te}_{20}$ isomers (b) CdTe nonisomers.

\begin{tabular}{|c|c|c|c|}
\hline & $\mathbf{G}_{\mathbf{1}}$ & $\mathbf{G}_{\mathbf{2}}$ & $\mathbf{G}_{\mathbf{3}}$ \\
\hline $\mathbf{a 1}$ & 0.128179 & 0.128179 & 0.743642 \\
\hline $\mathbf{a 2}$ & 0.333333 & 0.333333 & 0.333333 \\
\hline $\mathbf{a 3}$ & 0.383509 & 0.383509 & 0.232981 \\
\hline $\mathbf{a 4}$ & 0.390643 & 0.390643 & 0.218713 \\
\hline $\mathbf{a 5}$ & 0.408324 & 0.408324 & 0.183353 \\
\hline $\mathbf{a 6}$ & 0.445645 & 0.445645 & 0.108709 \\
\hline
\end{tabular}

(a)

\begin{tabular}{|c|c|c|c|}
\hline & $\mathbf{G}_{\mathbf{1}}$ & $\mathbf{G}_{\mathbf{2}}$ & $\mathbf{G}_{\mathbf{3}}$ \\
\hline b1 & 0.131062 & 0.131062 & 0.737876 \\
\hline b2 & 0.366790 & 0.366790 & 0.266420 \\
\hline b3 & 0.390643 & 0.390643 & 0.218713 \\
\hline b4 & 0.408324 & 0.408324 & 0.183353 \\
\hline b5 & 0.425645 & 0.425645 & 0.148710 \\
\hline b6 & 0.432427 & 0.432427 & 0.135146 \\
\hline
\end{tabular}

(b) 

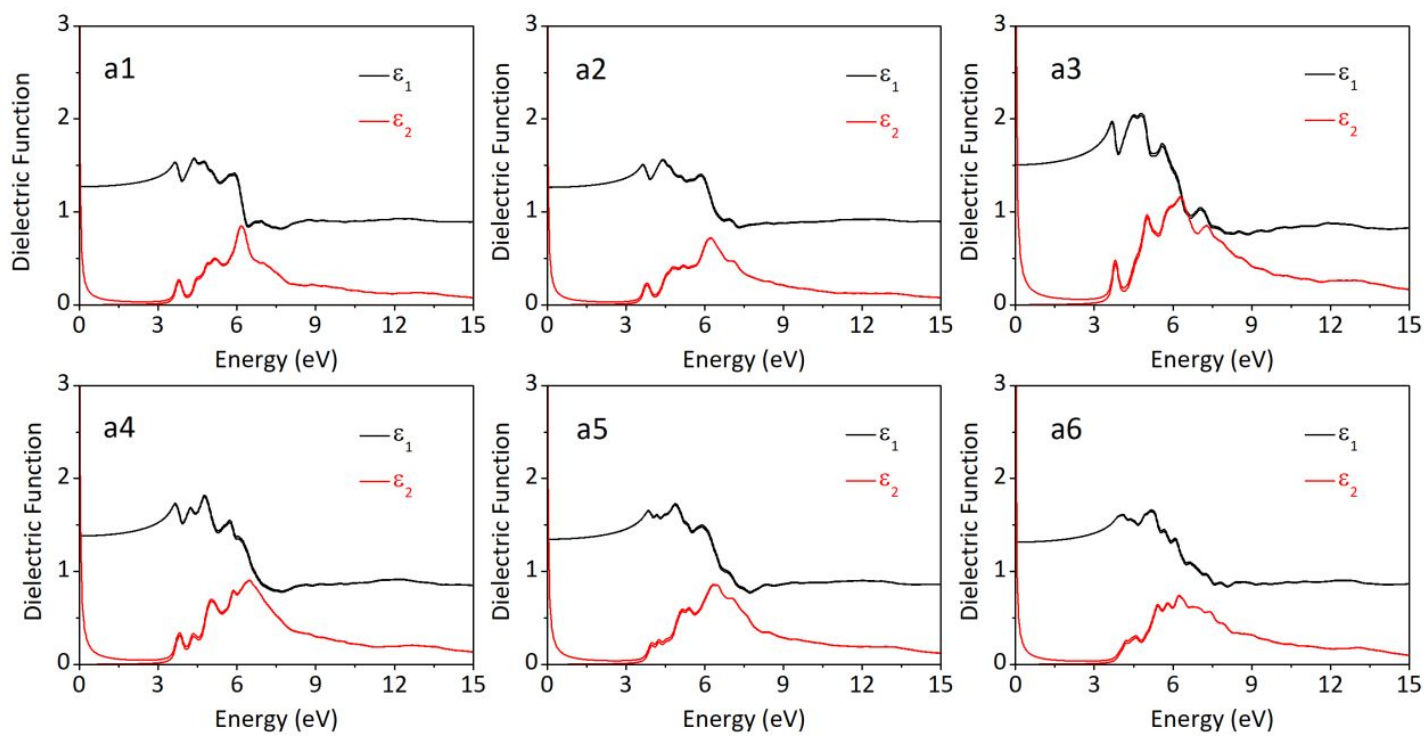

(a)
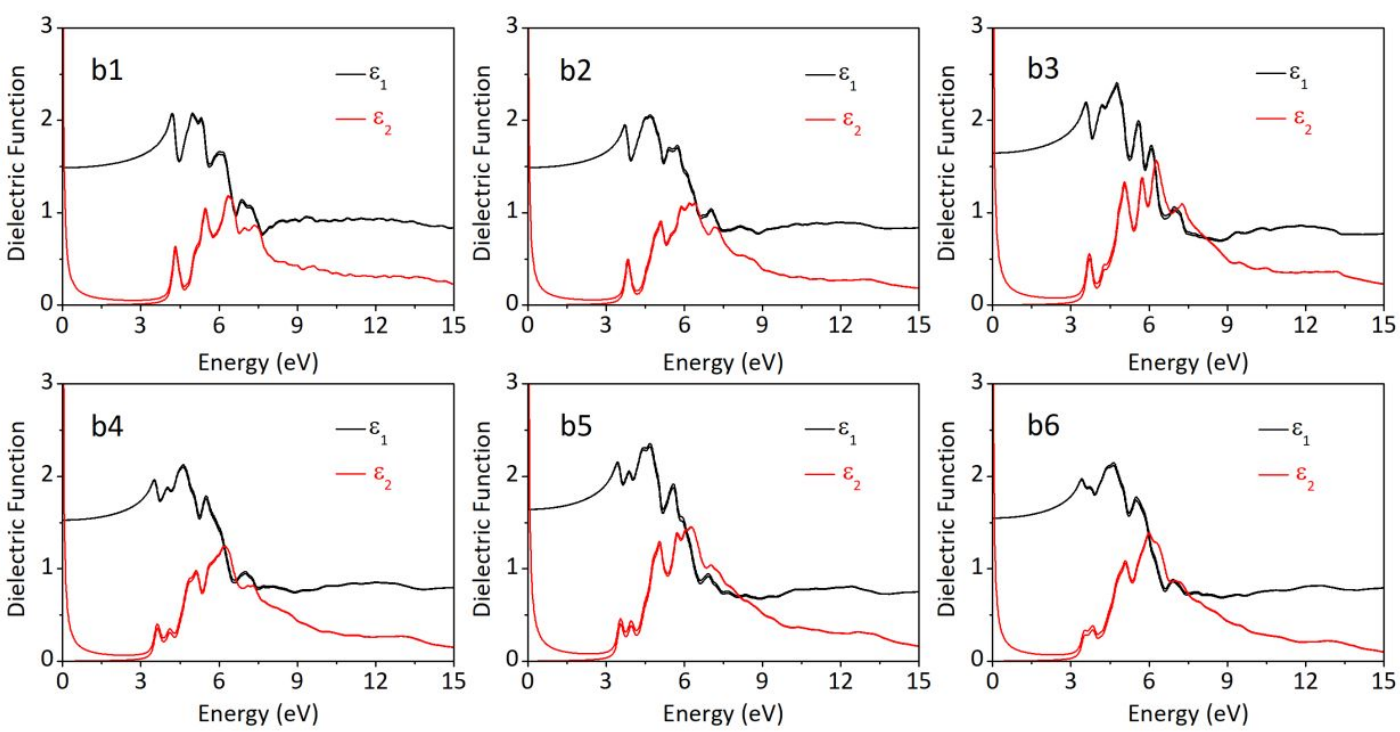

(b)

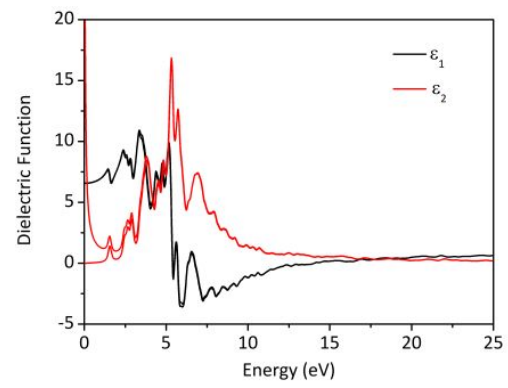

(c)

Figure S4. Dielectric function of (a) $\mathrm{Cd}_{37} \mathrm{Te}_{20}$ isomers (b) CdTe non-isomers (c) zincblende CdTe. $\varepsilon_{1}$ and $\varepsilon_{2}$ represent the real and imaginary part of dielectric function, 
respectively.

Table S2. Calculated square of $\operatorname{TDM}\left(\mu^{2}\right)$ and oscillator strengths $(f)$ for $(a) \mathrm{Cd}_{37} \mathrm{Te}_{20}$ isomers (b) CdTe non-isomers.

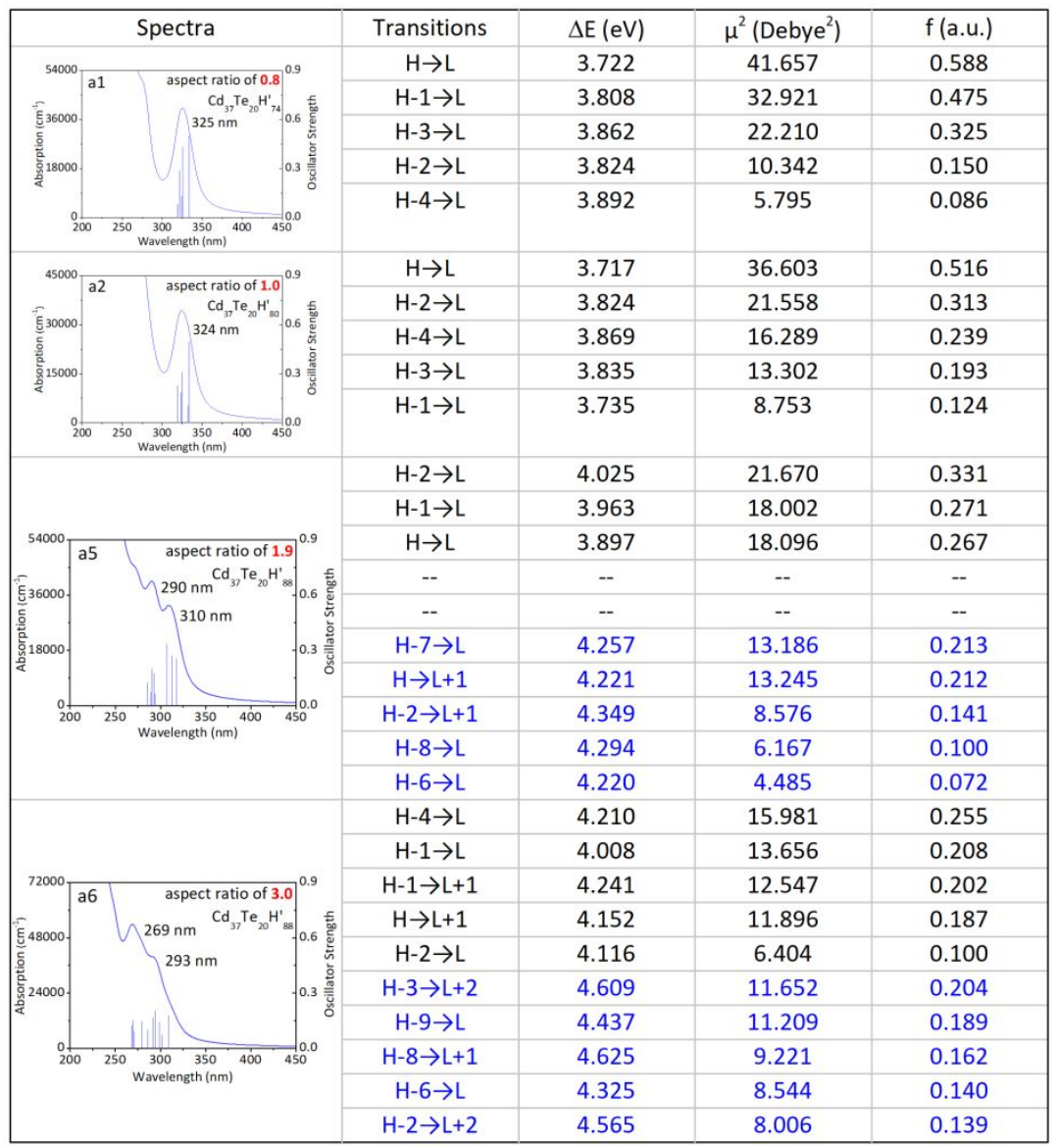

(a) 


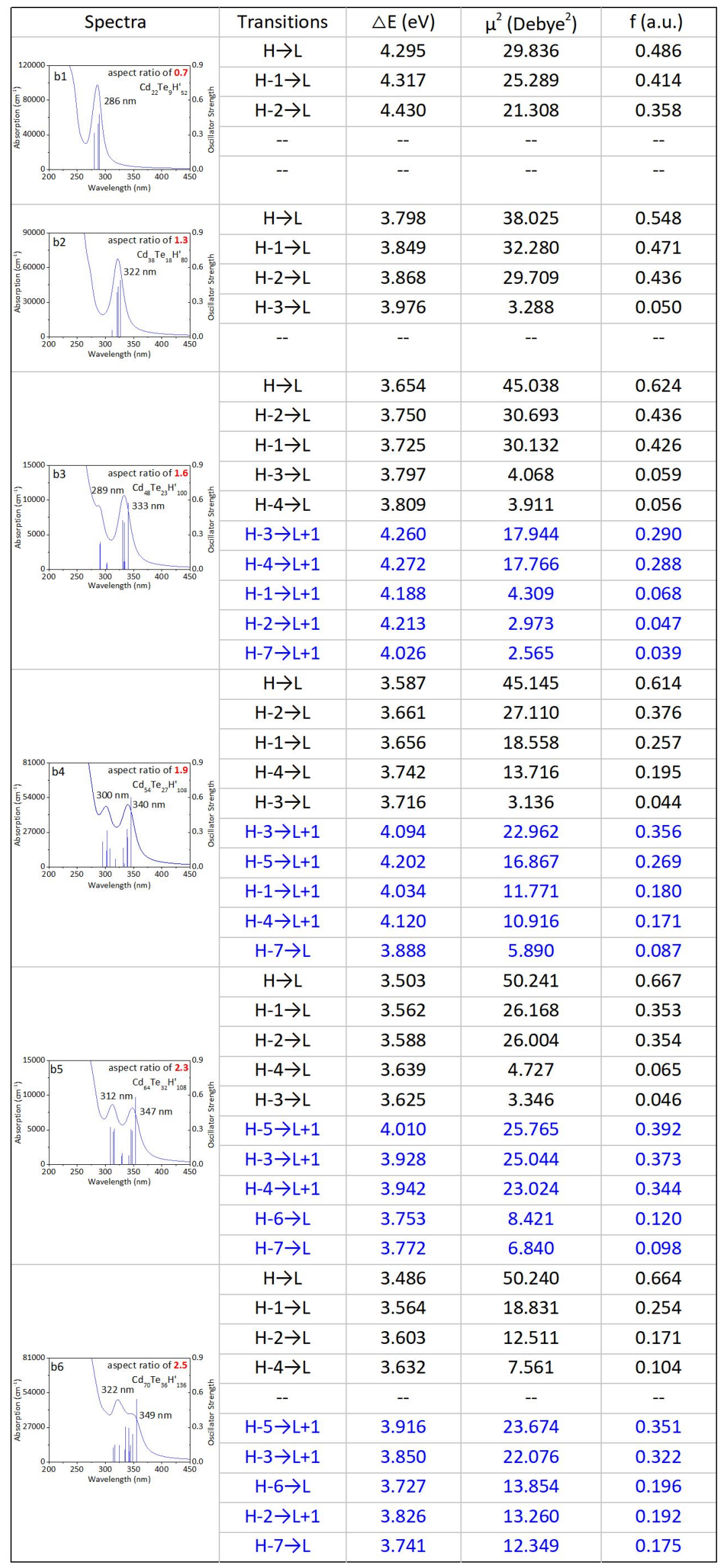


(b)

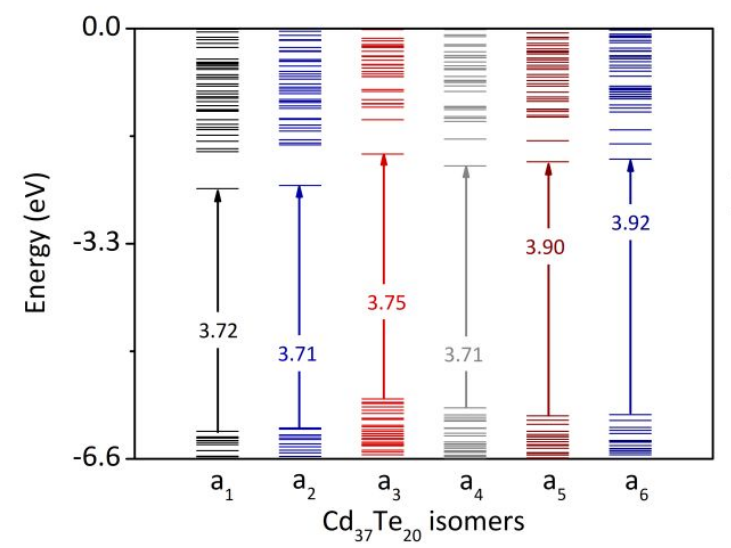

(a)

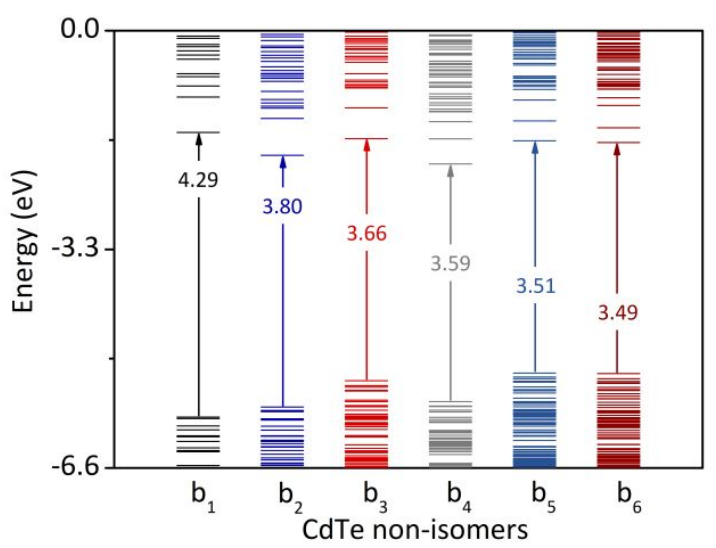

(b)

Figure S5. Energy level diagrams of (a) $\mathrm{Cd}_{37} \mathrm{Te}_{20}$ isomers and (b) CdTe non-isomers, in which KS HOMO-LUMO differences are marked. 
Table S3. The first twenty results for square of TDM $\left(\mu^{2}\right)$ and oscillator strengths $(f)$ in (a) a3 and (b) a4. (above $250 \mathrm{~nm}$, below $4.96 \mathrm{eV}$ )

\begin{tabular}{|c|c|c|c|}
\hline Transitions & $\Delta E(e V)$ & $\mu^{2}\left(\right.$ Debye $\left.^{2}\right)$ & $f($ a.u. $)$ \\
\hline$H \rightarrow L$ & 3.754 & 40.462 & 0.576 \\
\hline$H-2 \rightarrow L$ & 3.824 & 28.109 & 0.407 \\
\hline$H-6 \rightarrow L+1$ & 4.579 & 17.430 & 0.303 \\
\hline$H-1 \rightarrow L$ & 3.806 & 20.020 & 0.289 \\
\hline$H-3 \rightarrow L$ & 3.876 & 13.024 & 0.191 \\
\hline$H-4 \rightarrow L+1$ & 4.456 & 10.758 & 0.182 \\
\hline$H-11 \rightarrow L+1$ & 4.782 & 7.990 & 0.145 \\
\hline$H-7 \rightarrow L+3$ & 4.841 & 7.874 & 0.145 \\
\hline$H-2 \rightarrow L+2$ & 4.545 & 7.208 & 0.124 \\
\hline$H-3 \rightarrow L+1$ & 4.404 & 7.248 & 0.121 \\
\hline$H-1 \rightarrow L+7$ & 4.796 & 5.176 & 0.094 \\
\hline$H-4 \rightarrow L+5$ & 4.764 & 5.141 & 0.093 \\
\hline$H-4 \rightarrow L+4$ & 4.707 & 4.882 & 0.087 \\
\hline$H-5 \rightarrow L+4$ & 4.753 & 4.056 & 0.073 \\
\hline$H-1 \rightarrow L+1$ & 4.334 & 4.159 & 0.068 \\
\hline$H-3 \rightarrow L+4$ & 4.655 & 3.697 & 0.065 \\
\hline$H-3 \rightarrow L+3$ & 4.647 & 3.637 & 0.064 \\
\hline$H-5 \rightarrow L+3$ & 4.746 & 3.493 & 0.063 \\
\hline$H \rightarrow L+2$ & 4.476 & 3.063 & 0.052 \\
\hline$H-7 \rightarrow L+1$ & 4.598 & 2.921 & 0.051 \\
\hline
\end{tabular}

(a)

\begin{tabular}{|c|c|c|c|}
\hline Transitions & $\Delta E(e V)$ & $\mu^{2}\left(\right.$ Debye $\left.^{2}\right)$ & $f($ a.u. $)$ \\
\hline$H \rightarrow L$ & 3.713 & 35.941 & 0.506 \\
\hline$H-2 \rightarrow L$ & 3.857 & 29.573 & 0.432 \\
\hline$H-1 \rightarrow L$ & 3.818 & 24.997 & 0.362 \\
\hline$H-3 \rightarrow L+1$ & 4.304 & 21.199 & 0.346 \\
\hline$H-5 \rightarrow L+1$ & 4.431 & 17.506 & 0.294 \\
\hline$H-4 \rightarrow L+1$ & 4.329 & 14.111 & 0.232 \\
\hline$H-3 \rightarrow L+7$ & 4.802 & 8.420 & 0.153 \\
\hline$H-4 \rightarrow L$ & 3.916 & 9.900 & 0.147 \\
\hline$H-1 \rightarrow L+1$ & 4.231 & 7.484 & 0.120 \\
\hline$H-7 \rightarrow L+2$ & 4.783 & 6.570 & 0.119 \\
\hline$H-8 \rightarrow L+2$ & 4.821 & 5.947 & 0.109 \\
\hline$H-5 \rightarrow L$ & 4.018 & 5.303 & 0.081 \\
\hline$H-7 \rightarrow L+3$ & 4.833 & 4.106 & 0.075 \\
\hline$H-1 \rightarrow L+2$ & 4.499 & 4.307 & 0.073 \\
\hline$H-2 \rightarrow L+1$ & 4.270 & 3.328 & 0.054 \\
\hline$H-4 \rightarrow L+6$ & 4.808 & 2.945 & 0.054 \\
\hline$H \rightarrow L+4$ & 4.471 & 2.827 & 0.048 \\
\hline$H-2 \rightarrow L+4$ & 4.615 & 2.581 & 0.045 \\
\hline$H-5 \rightarrow L+4$ & 4.776 & 1.908 & 0.035 \\
\hline$H \rightarrow L+1$ & 4.126 & 2.185 & 0.034 \\
\hline
\end{tabular}


(b)
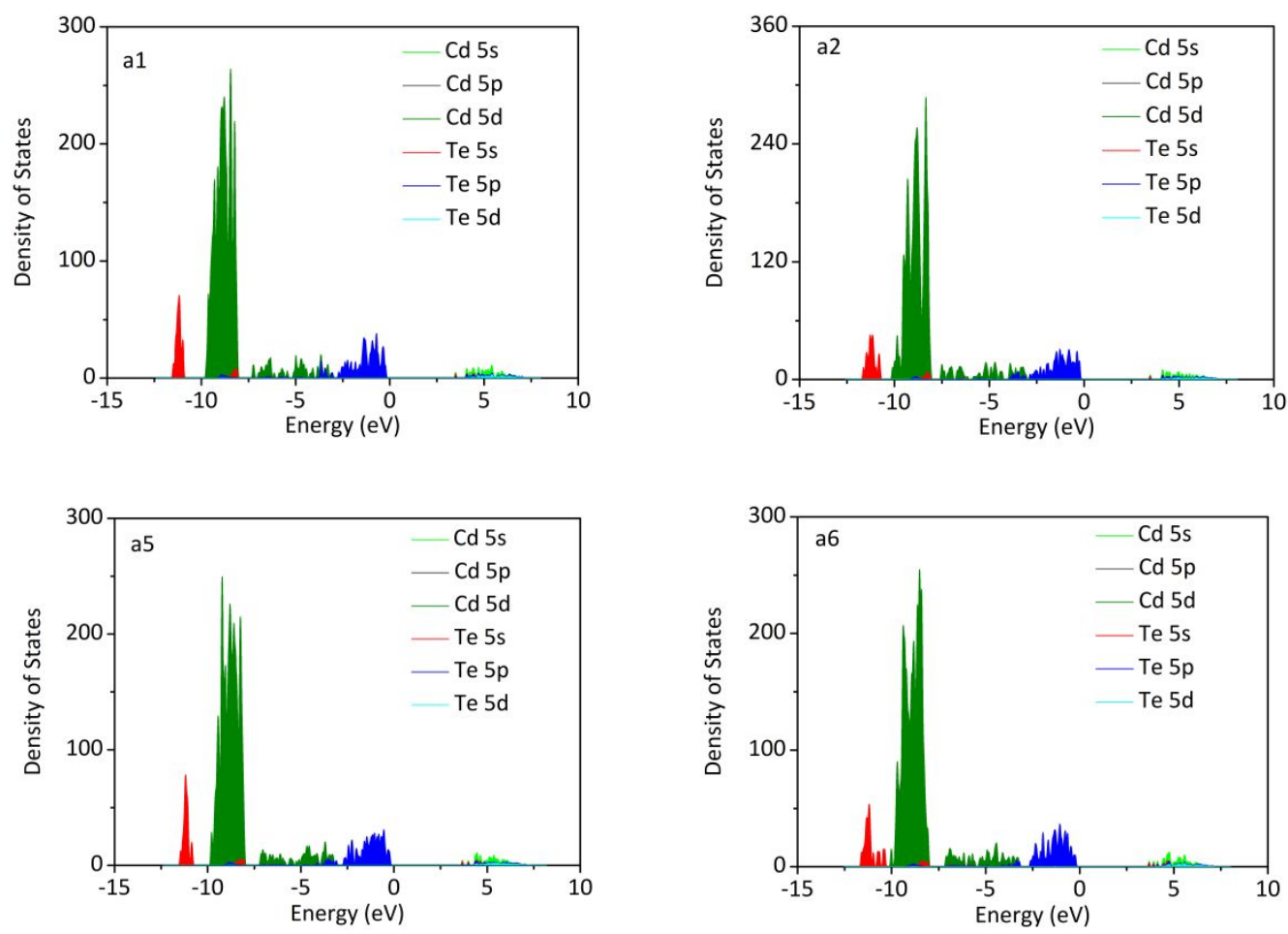

(a)
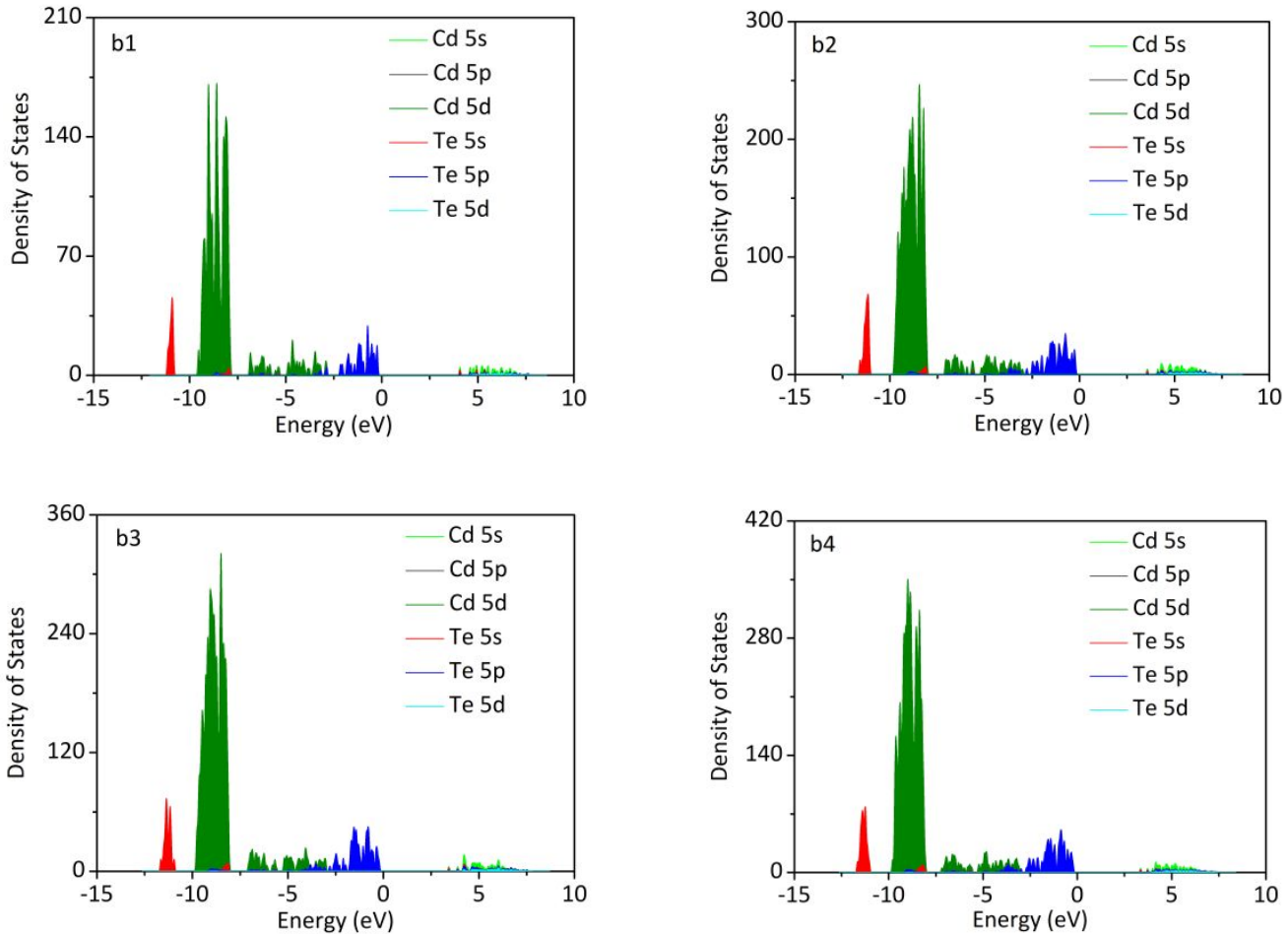

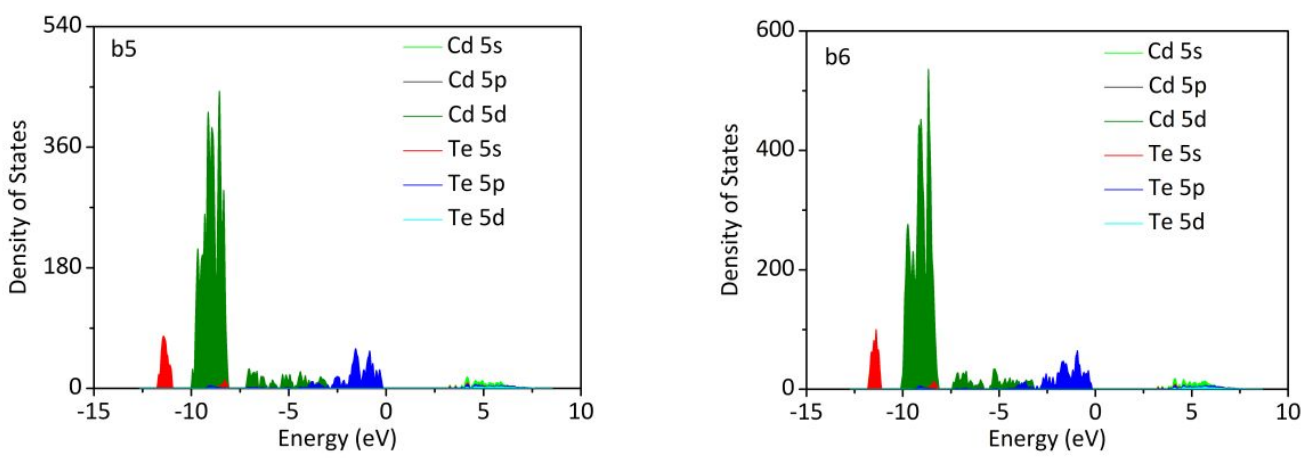

(b)

Figure S6. Density of states for (a) $\mathrm{Cd}_{37} \mathrm{Te}_{20}$ isomers (b) CdTe non-isomers. 


\section{REFERENCES}

(1) Gary, D. C.; Flowers, S. E.; Kaminsky, W.; Petrone, A.; Li, X.; Cossairt, B. M. SingleCrystal and Electronic Structure of a $1.3 \mathrm{~nm}$ Indium Phosphide Nanocluster. J. Am. Chem. Soc. 2016, 138, 1510-1513.

(2) Shen, Q.; Luan, C.; Rowell, N.; Zhang, M.; Wang, K.; Willis, M.; Chen, X.; Yu, K. Reversible Transformations at Room Temperature among Three Types of CdTe Magic-Size Clusters. Inorg. Chem. 2021, 60, 4243-4251. 